\title{
THE SPECTRAL CHARACTERIZATION OF NORMAL HYPERBOLICITY
}

\begin{abstract}
RICHARD SWANSON
ABSTRACT. In many cases hyperbolicity in dynamical systems can be expressed in terms of the spectrum of some canonically associated linear operator; e.g., the linearization at a fixed point. Such a characterization is known for Anosov diffeomorphisms and flows. We construct a vector bundle map, based on the tensor product, whose spectrum is decisive for detecting the normal hyperbolicity of a flow or diffeomorphism at an invariant manifold. This resolves a problem raised by Hirsch, Pugh and Shub. In the case of flows, our operator admits an infinitesimal formulation, which allows us to prove that normally hyperbolic systems are stable under reparameterization in many cases.
\end{abstract}

1. Introduction and main results. In Hirsch, Pugh and Shub [3], a study is made of dynamical systems on smooth manifolds which are normally hyperbolic at a fixed invariant submanifold. In the same treatment, several competing notions of normal hyperbolicity are introduced and contrasted. The weakest notion, "eventual relative normal hyperbolicity", defined below, is then shown to suffice to imply uniqueness and stability for the strongly stable and unstable laminations in the vicinity of the invariant submanifold. By a result of Mãne [4], it is known that eventual relative normal hyperbolicity is the optimal formulation; for any weaker definition, the invariant manifold is not smoothly stable under a perturbation of the dynamical system. Thus, we shall simply refer to eventual relative hyperbolicity as normal hyperbolicity.

Generally speaking, hyperbolicity in dynamical systems can be expressed in terms of the spectral features of associated linear maps, e.g. the linearization at a fixed point. For Anosov diffeomorphisms, J. Mather [5] gave a spectral characterization (and for flows, see [2]).

It is natural, then, to seek a spectral property equivalent to normal hyperbolicity -a problem raised in [3]. Specifically, one would like an operator, "canonically associated" to a diffeomorphism or flow, which is hyperbolic iff the dynamical system $\left(f\right.$ or $f_{t}$ ) is normally hyperbolic. Moreover, one would prefer that (1) no a priori requirement of an invariant normal bundle be imposed, and (2) the above canonical operator arise in a natural way from a vector bundle map. The tensor map, defined below, will satisfy these requirements.

Received by the editors August 11, 1982.

1980 Mathematics Subject Classification. Primary 58F15.

Key words and phrases. Normally hyperbolic, invariant manifolds, vector bundle flows.

(C) 1983 American Mathematical Society $0002-9939 / 83 \$ 1.00+\$ .25$ per page 
1.1. Definition. Given a $C^{1}$-diffeomorphism $f: M \rightarrow M$ on a smooth Riemannian manifold $M$, a compact invariant manifold $P$ of $f$ is normally hyperbolic at $P$ if the tangent bundle of $M$ restricted to $P, T M \mid P$, has a continuous splitting $T M \mid P=T P+$ $N^{s} P+N^{u} P$ where $T P$ is the tangent bundle of $P$, and $N^{s} P, N^{u} P$ are Tf-invariant subbundles of TM|P such that the bundle maps $(P f)_{x}=(T f)_{x} \mid(T P)_{x},\left(N^{u} f\right)_{x}=$ $(T f)_{x} \mid\left(N^{u} P\right)_{x}$, and $\left(N^{s} f\right)_{x}=(T f)_{x} \mid\left(N^{s} P\right)_{x}$ satisfy the estimates

(i) $\left\|\left(N^{s} f\right)_{x}^{n}\right\| \leqslant K \lambda^{n}$,

(ii) $\left\|\left(N^{u} f\right)_{x}^{-n}\right\| \leqslant K \lambda^{n}$,

(iii) $\left\|(P f)_{f^{n}(x)}^{-n}\right\| \cdot\left\|\left(N^{s} f\right)_{x}^{n}\right\| \leqslant K \lambda^{n}$, and

(iv) $\left\|(P f)_{x}^{n}\right\| \cdot\left\|\left(N^{u} f\right)_{f^{n}(x)}^{-n}\right\| \leqslant K \lambda^{n}$,

for constants $K>0,0<\lambda<1$, and all $x \in P$, all $n \in Z^{+}$. For a $C^{1}$-flow $f_{t}$ on $M$, with $P$ invariant, we shall call $P$ normally hyperbolic iff any particular map $f_{t}$ is normally hyperbolic, in which case all maps $f_{s}, s \in \mathbf{R}, s \neq 0$, are normally hyperbolic.

1.2. Definitions. $A$ Banach bundle $E$ over a p-manifold $P$ is a topological vector bundle whose fibers $E_{x}$ are isomorphic to a fixed model Banach space $E^{0}$ for all $x \in P$. The chart transfer maps induce Banach space isomorphisms on fibers (see Akin [1] for a categorical treatment of Banach bundles.) A Finsler on $E$ is an assignment of a norm \|\|$_{x}$ to each fiber $E_{x}$, compatible with its Banach space topology, and continuous in the sense that its representation in any locally trivializing chart is continuous (e.g. [1]). An automorphism $F=(F, f)$ of the vector bundle $E$ is a fiber-preserving homeomorphism which is linear on each fiber $E_{x}, x \in P$, and covers the homeomorphism $f: P \rightarrow P$, as in the diagram:

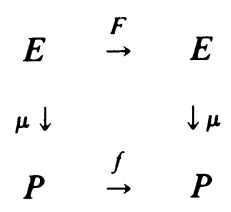

Thus, we require that the fiber map norms (from the Finsler), given by $\left\|F_{x}\right\|=\left\|F \mid E_{x}\right\|$ and $\left\|F_{x}^{-1}\right\|=\left\|F^{-1} \mid E_{x}\right\|$, be uniformly bounded in $x$.

An automorphism $F$ is hyperbolic on $E$, if the bundle $E$ splits as a continuous Whitney sum of two Banach subbundles $E=E^{s}+E^{u}$, such that, for some integer $n>0$,

$$
\left\|F_{x}^{n} \mid E_{x}^{s}\right\|<1 \text { and }\left\|F_{x}^{-n} \mid E_{x}^{u}\right\|<1
$$

for all $x \in P$. Here, $F^{n}=\left(F^{n}, f^{n}\right)$ is the $n$-fold composition of $F$ with itself. The fiber maps $F_{x}$ are always assumed to be invertible.

The next proposition gives a more intrinsic formulation of normal hyperbolicity. Continuing with the setting of a map $f: M \rightarrow M$ with a smooth invariant manifold $P$, we will not assume a priori the existence of an invariant normal bundle. Instead, form the quotient bundle $\mathscr{R} P=T_{P} M / T P$ over $P$ with induced map $\Re f$, leaving $P f$ as before. We can reorganize the definition of normal hyperbolicity as follows. 
1.3. Proposition [3]. For $(f, M, P)$ as above, normal hyperbolicity is equivalent to the conditions:

(a) $\Re f$ is a hyperbolic automorphism, with splitting $\Re P=\Re^{s} P+\Re^{u} P$.

(b) For some choice of Finsler on TP and $\Re P$, and some $n>0$,

(i) $\left\|\left(P f^{-n}\right)_{f^{n}(x)}\right\| \cdot\left\|\left(\mathscr{T}^{s} f^{n}\right)_{x}\right\|<1$ and

(ii) $\left\|\left(P f^{n}\right)_{x}\right\| \cdot\left\|\left(\Re^{u} f^{n}\right)_{x}^{-1}\right\|<1$.

The proof is immediate from the definitions. It is significant that (a) is equivalent to a simple spectral condition, which is worth stating in complete generality. Given a continuous bundle automorphism $(F, h)$ on the bundle $(E, P)$, with $h$ a homeomorphism of $P$, there is a natural linear operator $(F)_{\#}: \Gamma_{b}(E) \rightarrow \Gamma_{b}(E)$ on the space of bounded sections ( $E$ is Finslered) of $E, \Gamma_{b}(E)$, such that if $\eta \in \Gamma_{b}(E),\left(F_{\#} \eta\right)(x)=$ $(F \circ \eta)\left(h^{-1}(x)\right)$, for all $x \in P$.

The map $F_{\#}$ restricts continuously to the closed subspace $\Gamma_{c}(E)$ of continuous sections of $E$. The following proposition may be found in $[2,3]$.

1.4. Proposition. Let $\Gamma(E)$ denote either $\Gamma_{c}(E)$ or $\Gamma_{b}(E)$. Then $(F, h)$ is hyperbolic iff the spectrum of $F_{\#}: \Gamma(E) \rightarrow \Gamma(E)$ lies off the unit circle.

In particular, condition (a) in (1.3) holds iff $(\Re f)_{\#}$ has spectrum off the unit circle. It is condition (b) that appears to resist such a spectral pigeon hole. We now attempt to remedy this situation. Condition (b) is, essentially, a statement that the growth rates of $\Re f$ "nest", above and below, those of $P f$. To get at this behavior, first form the tensor product of $T^{*} P$, the contangent bundle of $P$, and $\Re P$, the quotient normal bundle. Thus, $T^{*} P \otimes \Re P$ is a bundle over $P$, with fiber at $x \in P$ given by $\left(T^{*} P\right)_{x} \otimes(\Re P)_{x}$. Tensor vectors (simple ones) are normed, as usual, so that $\|u \otimes v\|=\|u\| \cdot\|v\|$. Let $\Gamma_{b}\left(T^{*} P \otimes \Re P\right)$ denote the bounded sections. In what follows, let $P^{*} f$ denote the natural cotangent bundle map $T^{*}(f \mid P)$ which, by duality, covers the inverse map $f^{-1}$. Therefore, $\left(P^{*} f\right)^{-1}=P^{*} f^{-1}$ covers $f$. A necessary condition for normal hyperbolicity is given by our next result.

1.5. Proposition. If $(f, M, P)$ is normally hyperbolic at $P$, then $P^{*} f^{-1} \otimes \Re f$ is a hyperbolic automorphism. In particular, the associated map $\left(P^{*} f^{-1} \otimes \Re f\right)_{\#}$ acting on $\Gamma_{b}\left(T^{*} P \otimes \Re P\right)$ has spectrum off the unit circle.

Proof. Split $T^{*} P \otimes \Re P$ as a sum of $E^{s}=T^{*} P \otimes \Re^{s} P$ and $E^{u}=T^{*} P \otimes \Re^{u} P$. Note that $P^{*} f^{-1}$ is a vector bundle map covering $f$, and with respect to a given Finsler on $T P$ and $\Re P$, which fiberwise induces one on $T^{*} P$, we have, for all $x \in P$,

$$
\left\|\left(P f^{-n}\right)_{f^{n} x}\right\|=\left\|\left(P f^{-n}\right)_{x}^{*}\right\|=\left\|\left(P^{*} f^{-1}\right)_{x}^{n}\right\|,
$$

giving, by condition (i) in Proposition 1.3,

$$
\left\|\left(P^{*} f^{-1}\right)_{x}^{n}\right\| \cdot\left\|\left(\Re^{s} f\right)_{x}^{n}\right\|<1 \text { or }\left\|\left(P^{*} f^{-1} \otimes \Re^{s} f\right)_{x}^{n}\right\|<1 .
$$

From (ii) in (1.3), we obtain $\left\|\left(P^{*} f^{-1} \otimes \mathcal{T}^{u} f\right)_{x}^{-n}\right\|<1$. Thus, $E^{s}+E^{u}$ is the desired splitting. 
1.6. Remarks. The converse is easily seen to be false; e.g., $P^{*} f^{-1} \otimes \Re f$ can be hyperbolic when $\Re f$ is not. Also, $\left(P^{*} f^{-1} \otimes \Re f\right)_{\#}$ does not equal $\left(P^{*} f^{-1}\right)_{\#} \otimes$ $(\Re f)_{\#}$; in fact, the former is a quotient map of the latter.

Thus, we seek now to strengthen the tensor hyperbolicity condition above. Since the hyperbolicity of $\Re f$ is already a spectral property, we shall assume that $\Re P$ splits into the stable and unstable parts $\Re^{s} P+\Re^{u} P$ with $\pi_{s}$ and $\pi_{u}$, the corresponding bundle projections. For values of $\mu, 0 \leqslant \mu<\infty$, define the bundle isomorphisms over the identity, $A_{\mu}=e^{-\mu} \pi_{s}+e^{\mu} \pi_{u}$.

Finally, define the family of vector bundle maps $F_{\mu}$ on $T^{*} P \otimes \Re P$, such that

$$
F_{\mu}=P^{*} f^{-1} \otimes A_{\mu} \Re f, \quad \mu \geqslant 0 .
$$

1.7. Definition. Let us call the family $\left\{F_{\mu}: 0 \leqslant \mu<\infty\right\}$ uniformly hyperbolic if for each $\mu, 0 \leqslant \mu<\infty$, the spectrum of $\left(F_{\mu}\right)_{\#}$ lies outside a fixed annulus, independent of $\mu$, containing the unit circle in its interior.

A simple expedient allows us to lift uniform hyperbolicity to the hyperbolicity of a single Banach bundle map. Form a bundle $B$ over $P$ whose fiber at $x$ is given by

$$
B_{x}=\left\{a:[0, \infty) \rightarrow\left(T^{*} P\right)_{x} \otimes(\Re P)_{x} \mid a \text { is bounded }\right\},
$$

i.e., each fiber is a Banach space of bounded curves, which is Finslered so that if $a_{x} \in B_{x},\left\|a_{x}\right\|=\sup \left\{\|a(\mu)\|_{x}: 0 \leqslant \mu<\infty\right\}$. The family $F_{\mu}$ induces a lift $B F: B \rightarrow B$ such that

$$
[B F(a)](\mu)=F_{\mu}(a(\mu)) .
$$

Clearly, $(B F, f)$ is a vector bundle automorphism on $(B, P)$. We can now state and prove our main theorem.

1.9. ThEOREM. Given a diffeomorphism $f: M \rightarrow M$, having the invariant smooth submanifold $P \subseteq M$, such that the quotient map $\Re f$ is hyperbolic on $T_{P} M / T P$, then $f$ is normally hyperbolic at $P$ if and only if the canonical lift automorphism $B F: B \rightarrow B$ is hyperbolic.

1.10. Corollary. For smooth flows $f_{t}$, such that the time-one map $f_{1}$ satisfies the hypotheses of the theorem (with $f=f_{1}$ ), the one-parameter family of lifts $B F_{t}: B \rightarrow B$ is hyperbolic, for each $t \neq 0$, if and only if the flow is normally hyperbolic at $P$.

Proof. Suppose $f$ is normally hyperbolic at $P$. We shall show that this assures the uniform hyperbolicity of the family $F_{\mu}$. Indeed, split $T^{*} P \otimes \Re P$ as $\left(T^{*} P \otimes \Re^{s} P\right)+$ $\left(T^{*} P \otimes \Re^{u} P\right)$, which for simplicity, we write as $E^{s}+E^{u}$. Then for some $n>0$,

$$
\left\|F_{\mu}^{n} \mid E_{x}^{s}\right\|=e^{-\mu}\left\|\left(P^{*} f^{-1} \otimes \Re^{s} f\right)_{x}^{n}\right\|=e^{-\mu}\left\|\left(P^{*} f^{-n}\right)_{x}\right\| \cdot\left\|\left(\Re^{s} f^{n}\right)_{x}\right\|<e^{-\mu} \leqslant 1,
$$

and similarly, $\left\|F_{\mu}^{-n} \mid E_{x}^{u}\right\|<e^{-\mu} \leqslant 1$. Conversely, suppose $\left\{F_{\mu}\right\}$ is uniformly hyperbolic for $0 \leqslant \mu<\infty$. Thus, a priori, for each $\mu$, the bundle $T^{*} P \otimes \Re P$ splits as a sum $E_{\mu}^{s}+E_{\mu}^{u}$, with $F_{\mu}$ contracting $E_{\mu}^{s}$ and $F_{\mu}^{-1}$ contracting $E_{\mu}^{u}$. For each $\mu \geqslant 0$, the decomposition

$$
H^{s}+H^{u}=\left(T^{*} P \otimes \Re^{s} P\right)+\left(T^{*} P \otimes \Re^{u} P\right)
$$


is also $F_{\mu}$-invariant. Since $F_{\mu}$ is hyperbolic and $H^{s}$ is complemented by an $F_{\mu}$ invariant subbundle, $F_{\mu} \mid H^{s}$ is hyperbolic. Form the operator $\left(F_{\mu} \mid H^{s}\right)_{\#}$ on the section space $\Gamma\left(H^{s}\right)$. Evidently, the spectrum of $\left(F_{\mu} \mid H^{s}\right)_{\#}$ is given by

$$
\sigma\left(F_{\mu} \mid H^{s}\right)_{\#}=e^{-\mu} \sigma\left(F_{0} \mid H^{s}\right)_{\#} .
$$

But, if the spectral radius of $\left(F_{0} \mid H^{s}\right)_{\#}$ is $r_{0}$, there exists a $\mu \geqslant 0$ with $e^{-\mu} r_{0}=1$, unless $r_{0}<1$. Since $\sigma\left(F_{\mu}\right)_{\#}$ lies off the unit circle, for $\mu \geqslant 0$, we infer that $r_{0}<1$ and $\left(F_{0} \mid H^{s}\right)_{\#}$ is a contraction. In particular $H^{s} \subseteq E_{0}^{s}$. A similar argument with $F_{\mu}^{-1}$, gives $H^{u} \subseteq E_{0}^{u}$ and, by finite dimensions, we have $E_{0}^{s}=H^{s}, E_{0}^{u}=H^{u}$. Repeating this process with $F_{\mu_{0}}$ in place of $F_{0}$, for any fixed $\mu_{0} \geqslant 0$, leads to the conclusion that for all $\mu \geqslant 0$,

$$
E_{\mu}^{s}=T^{*} P \otimes \mathcal{K}^{s} P \quad \text { and } \quad E_{\mu}^{n}=T^{*} P \otimes \Re^{u} P .
$$

For this splitting, the hyperbolicity of $F_{0}$ yields the desired normal hyperbolicity.

It remains to prove that $\left\{F_{\mu}\right\}$ is uniformly hyperbolic iff a certain hyperbolic bundle map exists. Let $B F$ and $B$ be as defined above. Assume $\left\{F_{\mu}\right\}$ is uniformly hyperbolic, and split the bundle $B$ as $B^{s}+B^{u}$ with $B_{x}^{s}=\left\{a \in B_{x}: a(\mu) \in\left(T^{*} P\right)_{x} \otimes\right.$ $\left.\left(\mathcal{T}^{s} P\right)_{x}, \mu \geqslant 0\right\}$ and $B_{x}^{u}=\left\{a \in B_{x}: a(\mu) \in\left(T^{*} P\right)_{x} \otimes\left(\mathcal{T}^{u} P\right)_{x}, \mu \geqslant 0\right\}$. Then, it is easily verified that $B F$ is hyperbolic with respect to this continuous splitting.

Now, assume $B F$ is hyperbolic on $B$. Recall that the fibers $B_{x}$ consist of bounded maps from the half-line $(\mu \geqslant 0)$ into $\left(T^{*} P\right)_{x} \otimes(\mathcal{N} P)_{x}$.

Define the subbundles $B_{\mu}$ of $B$ with fibers $\left(B_{\mu}\right)_{x}=\left\{a \in B_{x}: a(\nu)=0\right.$ for $\left.\nu \neq \mu\right\}$. Thus, $B_{\mu}$ is a copy of $T^{*} P \otimes \Re P$ for each $\mu \geqslant 0$, which is $B F$-invariant and complemented by the $B F$-invariant bundle $D_{\mu}$ with fiber at $x,\left(D_{\mu}\right)_{x}=\left\{a \in B_{x}\right.$ : $a(\mu)=0\}$.

Hence, $B F \mid B_{\mu}$ is hyperbolic, for each $\mu \geqslant 0$, with uniformly hyperbolic estimates, independent of $\mu$. Finally, $B F \mid B_{\mu}$ acting on $B_{\mu}$ over $f$ is obviously bundle-equivalent to $F_{\mu}$ acting on $T^{*} P \otimes \Re P$, which completes our proof.

1.11. Remark. Perhaps (1.9) is best understood as equating normal hyperbolicity to the hyperbolicity of a pair of vector bundle maps $(\Re f, f)$ and $(B F, f)$ such that the map $B F$ is defined once the hyperbolicity of $\Re f$ is established. In applications, as we shall see below, it is often easier to replace the hyperbolicity of $(B F, f)$ by the uniform hyperbolicity of the family $\left\{F_{\mu}\right\}$.

2. Infinitesimal normal hyperbolicity in flows. Suppose $X$ is a smooth vector field on $M$ which generates a normally hyperbolic flow at the invariant smooth manifold $P$, i.e. each time- $t$-map $f_{t}$ for $t \neq 0$, of the flow is normally hyperbolic at $P$. It will be useful to form the vector bundle flow $F_{t}=P^{*} f_{t}^{-1} \otimes \Re f_{t}$ acting on $T^{*} P \otimes \Re P$, which induces the group of linear operators $\left(F_{t}\right)_{\#}$ acting on the Banach section space $\Gamma_{c}\left(T^{*} P \otimes \Re P\right)$. The group $\left(F_{t}\right)_{\#}$ is a strongly-continuous semigroup with infinitesimal generator $\mathscr{D}_{X}$, defined on a dense subspace of $\Gamma_{c}\left(T^{*} P \otimes \Re P\right)$. To see the form that $\mathscr{D}_{X}$ takes, choose a section of $T^{*} P \otimes \Re P$ of the special form $\omega \otimes Z$, where $\omega$ is a continuous section of $T^{*} P$ and $Z$ is a continuous section of $\Re P$. In fact, the totality of continuous sections of $T^{*} P \otimes \mathfrak{N} P$ is given by the uniform closure of 
finite sums of the form $\Sigma \omega_{i}(x) \otimes Z_{i}(x)$; by the Stone-Weierstrass theorem. Therefore,

$$
\mathscr{D}_{X}(\omega \otimes Z)(x)=\left.\frac{d}{d t}\right|_{t=0}\left\{P^{*} f_{-t} \omega\left(f_{-t} x\right) \otimes \Re f_{t} Z\left(f_{-t} x\right)\right\},
$$

which by Leibniz' rule becomes

$$
\left[\left(\varrho_{X} \omega\right)(x) \otimes Z(x)\right]+\left[\omega(x) \otimes\left(L_{X} Z\right)(x)\right]
$$

where $\varrho_{X}$ denotes Lie differentiation of one-forms and $L_{X}$ is the ordinary Jacobi bracket $L_{X} Y=[Y, X]$, interpreted modulo sections of $T P$, since $\Re P=T_{P} M / T P$. We may, therefore, express $\mathscr{D}_{X}$ as $\mathscr{D}_{X}=\left(\varrho_{X} \otimes I\right)+\left(I \otimes L_{X}\right)$, a sum of commuting differential opertors. For the associated family of operators $\left(F_{t}\right)_{\mu}=P^{*} f_{-t} \otimes A_{\mu} \Re f_{t}$, employed in the proof of Theorem 1.9, the generators have the form, for $\mu \geqslant 0$,

$$
\left(\mathscr{D}_{X}\right)_{\mu}=\left(\varrho_{X} \otimes A_{\mu} I\right)+\left(I \otimes A_{\mu} L_{X}\right) \text {. }
$$

It can be shown that $\left(\mathscr{D}_{X}\right)_{\mu}$ has spectrum off the imaginary axis iff $\left(F_{t}\right)_{\mu}$ forms a semigroup in $t$, of hyperbolic bundle maps [7]. This yields the infinitesimal version of Corollary 1.10:

2.1. Proposition. Assume that $\Re f_{1}$ is hyperbolic. The flow $f_{t}$ is normally hyperbolic at $P \subseteq M$ iff $\left(\mathscr{D}_{X}\right)_{\mu}$ has spectrum bounded (uniformly) off the imaginary axis, for $\mu \geqslant 0$.

If $X \neq 0$ on $P$, we can form the quotient bundle $T P /[X]$, with $[X]$ the line bundle spanned by $X$. Let $P f_{t}$ denote the quotient map of $P f_{t}$ applied to $T P /[X]$. The dual map $P^{*} f_{t}$ is defined naturally on the bundle $X^{\perp}=\left\{\omega_{x} \in T^{*} P \mid \omega_{x} X(x)=0\right\}$. Thus, $\mathcal{P}^{*} f_{t}^{-1} \otimes \Re f_{t}$ is simply a restriction of $P^{*} f_{t}^{-1} \otimes \mathfrak{N} f_{t}$ employed above.

The family of bundle maps $\left(F_{t}\right)_{\mu}$ restricts to the bundle $X^{\perp} \otimes \mathscr{N} P$, and is hyperbolic if $X$ is normally hyperbolic at $P$, with the obvious splitting $\left(X^{\perp} \otimes \Re^{s} P\right)$ $+\left(X^{\perp} \otimes \mathcal{K}^{u} P\right)$. Since $\left(F_{t}\right)_{\mu}$ is hyperbolic on $[X]^{*} \otimes \Re P=\left(T^{*} P / X^{\perp}\right) \otimes \Re(P$, the converse is easily seen to hold, and we can summarize these findings, as follows. Assume, again, that $\Re f$ is hyperbolic.

2.2. Proposition. If $X \neq 0$ on $P$, it is necessary and sufficient for normal hyperbolicity to require that the restriction of $\left(\mathscr{D}_{X}\right)_{\mu}$ to sections of $X^{\perp} \otimes \Re P$ have spectrum off the imaginary axis for $\mu \geqslant 0$.

We come now to the main result in this section, concerning reparameterization.

2.3. TheOREM. Suppose that the $C^{1}$ flow $X$ on $M$ is such that $X \mid P$ is nonsingular and generates a flow in $P$ with a dense set of nonperiodic points. If $X$ is normally hyperbolic at $P$, then $\phi X$ is normally hyperbolic at $P$ for any smooth $\phi: M \rightarrow \mathbf{R}, \phi>0$. Moreover, the quotient normal bundle splitting $\mathfrak{N} P=\mathfrak{N}^{s} P+\mathfrak{T}^{u} P$ persists under reparameterization.

Proof. We first show that $\Re^{s} P$ and $\Re^{u} P$ are unchanged with reparameterization. The normal semigroup $\left(\mathfrak{N} f_{t}\right)_{\#} Z(x)=\mathscr{N} f_{t} Z\left(f_{-t} x\right)$ has infinitesimal generator $L_{X}$, 
which denotes the Jacobi bracket of sections of $T M$ taken modulo sections of $T P$. By the hyperbolicity of $\Re f_{t}, L_{X}$ has spectrum disjoint from the imaginary axis. The sections of $\Re^{s} P$ correspond to the invariant subspace of $L_{X}$ whose spectrum lies in the strictly negative (real) half-plane; while, sections of $\mathscr{N}^{u} P$ arise from the spectral component of positive real part. These subspaces are uniquely determined. Reparameterization $Y=\phi X, \phi>0$, requires that we examine the operator $L_{Y}=L_{\phi X}$. It is standard fact that $L_{\phi X} Z=\phi L_{X} Z+Z \phi X$, and, taking $Z$ modulo vector fields tangent to $P$, means that we can excise the function multiple of $X$, leaving the relation $L_{\phi X} Z=\phi L_{X} Z$. Since $\phi(x)$ is real and positive, $\phi L_{X}$ is invertible whenever $L_{X}$ is, which also suffices to rule out any imaginary spectrum (in the generic case of a dense set of nonperiodic points) [2]. Then $\phi X$ generates a family of hyperbolic bundle maps on $\mathscr{N} P$ whenever the family $\mathcal{N} f_{t}$, derived from $X$, is hyperbolic. Moreover, the reparameterized vector field has the same contractive/expansive normal subbundle splitting.

Thus, the infinitesimal generators

$$
\left(\mathscr{D D}_{\phi X}\right)_{\mu}=\left(L_{\phi X} \otimes A_{\mu} I\right)+\left(I \otimes A_{\mu} L_{\phi X}\right),
$$

are defined for $\mu \geqslant 0$, and satisfy the relation

$$
\left(\mathscr{D}_{\phi X}\right)_{\mu}(\omega \otimes Z)=\phi\left(\cdot D_{X}\right)_{\mu}(\omega \otimes Z)+\omega(X) d \phi \otimes A_{\mu} Z .
$$

for simple tensors $\omega \otimes Z$. Hence, on the closure of sections $\Sigma\left(\omega_{i} \otimes Z_{i}\right)$ with $\omega_{i} X=0$ the operator $\left(\mathscr{D}_{\phi X}\right)_{\mu}=\phi\left(\mathscr{D}_{X}\right)_{\mu}$ is invertible, as desired, by Proposition 2.2 .

2.4. Remark. Actually, the hypothesis that $X \mid P$ be nonsingular can probably be removed via a local "partition-of-unity" argument. The same goes for the assumption that nonperiodic points are dense.

\section{REFERENCES}

1. E. Akin, The metric theory of Banach manifolds, Lecture Notes in Math., vol. 662, Springer-Verlag, New York, 1978.

2. C. Chicone and R. Swanson, Spectral theory for linearizations of dynamical systems, J. Differential Equations 40 (1981), 155-167.

3. M. W. Hirsch, C. C. Pugh and M. Shub, Invariant manifolds, Lecture Notes in Math., vol. 583, Springer-Verlag, Berlin, Heidelberg and New York, 1977.

4. R. Mãne, Persistent manifolds are normally hyperbolic, Bull. Amer. Math. Soc. 80 (1974), 90-91.

5. J. Mather, Characterization of Anosov diffeomorphisms, Indag. Math. 30 (1968), 479-483.

6. J. F. Selgrade, Isolated invariant sets for flows on vector bundles, Trans. Amer. Math. Soc. 203 (1975), 359-390.

7. R. Swanson, The spectrum of vector bundle flows with invariant subbundles, Proc. Amer. Math. Soc. 83 (1981), 141-145.

Department of Mathematical Sciences, Montana State University, Bozeman, Montana 59717 